\title{
NOTE ON THE JOINT SPECTRUM OF THE WIENER-HOPF OPERATORS
}

\author{
JAN JANAS 1
}

\begin{abstract}
J. Bunce has given [2] the definition of a joint approximate point spectrum of $p$-tuples of commuting operators in a complex Hilbert space. A. T. Dash after presentation of another type of a joint spectrum, has found this joint spectrum for $p$-tuples of analytic Toeplitz operators [4]. In this paper we will find a joint approximate point spectrum of $p$-tuples of noncommuting selfadjoint Wiener-Hopf operators and prove an inclusion for a joint spectrum of analytic matrix Toeplitz operators.
\end{abstract}

1. There are several types of joint spectra of $n$-commuting operators. Among others, an interesting concept of a joint spectrum was proposed by J. L. Taylor [9], but we will not consider it in this paper. In what follows we will need two types of joint spectra. The first type of joint spectrum we need was defined by J. Bunce [2]; it is the left joint spectrum. The second type of joint spectrum was proposed by F. Bonsall and I. Duncan [1]; it is the union of the left and right spectrum. Let us recall their definitions.

Let $L(H)$ be the algebra of all linear bounded operators in a complex Hilbert space $H$. Denote by $\widetilde{T}=\left(T_{1}, \cdots, T_{n}\right) n$-tuples of commuting operators in $H$. We have the following definition.

Definition 1. Let $\widetilde{T}$ be as above. The left (right) joint spectrum $\sigma_{l}(\tilde{T}) / \sigma_{r}(\widetilde{T})$ of $\tilde{T}$ is defined as a set of all $\lambda \in \mathbf{C}^{n}$ such that the family $\lambda_{i}-T_{i}$, $i=1, \cdots, n$, generates in the algebra $L(H)$ a proper left (right) ideal.

The definition of the joint approximate point spectrum reads as follows.

Definition 2. Let $\widetilde{T}$ be as in the Definition 1. The joint approximate point spectrum $\sigma_{\pi}(\widetilde{T})$ is defined as a set of all $\lambda \in \mathbf{C}^{n}$ such that there exists a sequence $f_{k} \in H,\left\|f_{k}\right\|=1$, for all $k$ and $\lim _{\sim} \rightarrow \infty\left\|\left(\lambda_{i}-T_{i}\right) f_{k}\right\|=0$ for $i=$ $1, \cdots, n$. It is well known that $\sigma_{\pi}(\widetilde{T})=\sigma_{l}(\tilde{T})$.

Remark 1. Definitions 1 and 2 also have sense for noncommuting family of operators.

Received by the editors March 25, 1974.

AMS (MOS) subject classifications (1970). Primary 47B35.

1 I take this opportunity to express my thanks to Professor R. G. Douglas for valuable suggestions during preparation of this paper. 
Let $\Delta$ be the open unit disc in the complex plane $\mathrm{C}$ and let $m$ be the normalized Lebesque measure on the borel subsets of the unit circle $\Gamma$. We denote by $\mathcal{L}^{2}$ the usual Hilbert space of $m$ square integrable functions on $\Gamma$. Let $H^{2}$ be the closure of polynomials in $\mathcal{L}^{2}$ and $H^{\infty}$ the weak-star closure of polynomials in $\mathcal{Q}^{\infty}$ (essentially bounded, $m$ measurable).

If $\varphi$ is in $\varrho^{\infty}$, we define the Toeplitz operator $T_{\varphi}$ on $H^{2}$ by

$$
T_{\varphi} f=P(\varphi \cdot f) \text { for } f \in H^{2}
$$

where $P$ is the projection of $\mathcal{Q}^{2}$ onto $H^{2}$.

If $\varphi$ is analytic then $T_{\varphi}$ is called the analytic Toeplitz operator. One can also define the matrix generalization of the Toeplitz operator [5]. Let us write it precisely.

For a natural number $n \geq 1$ we define the direct sum $\bigoplus_{i=1}^{n} H_{i}^{2}$ of $n$ copies of the Hardy space $H^{2}$. Let $\phi$ be a $n \times n$ matrix over $\mathcal{L}^{\infty}$. (We will write $\phi \in \mathscr{2}_{n \times n^{\circ}}^{\infty}$ )

Let $\hat{P}$ be the orthogonal projection of $\bigoplus_{i=1}^{n} \mathfrak{Q}_{i}^{2} \rightarrow \bigoplus_{i=1}^{n} H_{i}^{2}$. We define the matrix Toeplitz operator on $\bigoplus_{i=1}^{n} H_{i}^{2}$ by

$$
\hat{T}_{\phi} \hat{f}=\hat{P}(\phi \hat{f}) \text { for } \hat{f} \in \bigoplus_{i=1}^{n} H_{i}^{2} .
$$

If $\phi$ is a matrix whose entries $\varphi_{i j}$ are in $H^{\infty}$, we write $\phi \in H_{n \times n}^{\infty}$. In what follows we will use the natural isometry of $H^{2}$ and the space of all analytic functions in $\Delta$ with square summable Taylor series.

Now we will prove

Theorem 1. Let $\phi_{i} \in H_{n \times n}^{\infty}$ be analytic matrices for $i=1, \cdots, p$. Denote by $\hat{T}_{\phi_{i}}$ the corresponding Toeplitz operators. Then the following inclusion bolds true:

$$
\begin{aligned}
\sigma_{l}\left(\hat{T}_{\phi_{1}}, \cdots, \hat{T}_{\phi_{p}}\right) & \cup \sigma_{r}\left(\hat{T}_{\phi_{1}}, \cdots, \hat{T}_{\phi_{p}}\right) \\
& \subset\left\{\lambda \in \mathbf{C}^{p}, \inf _{\Delta} \sum_{i=1}^{p}\left|\operatorname{det}\left(\lambda_{i}-\phi_{i}(z)\right)\right|=0\right\}=Z .
\end{aligned}
$$

Proof. We will prove the implication:

$$
\lambda \notin Z \Rightarrow \lambda \notin \sigma_{l}\left(\hat{T}_{\phi_{1}}, \cdots, \hat{T}_{\phi_{p}}\right) \cup \sigma_{r}\left(\hat{T}_{\phi_{1}}, \cdots, \hat{T}_{\phi_{p}}\right) .
$$

Observe first that for $\lambda \notin Z$, there exists $\delta>0$ such that the following inequality is true:

$$
\inf _{\Delta}\left\{\sum_{i=1}^{p}\left\|\left(\lambda_{i}-\phi_{i}(z)\right) f\right\|, f \in \mathbf{C}^{n},\|f\|=1\right\} \geq \delta .
$$


If not, then there are sequences $\left\{f_{s}\right\} \subset \mathbf{C}^{n},\left\|f_{s}\right\|=1,\left\{z_{s}\right\} \subset \Delta$ such that

$$
\left\|\left(\lambda_{i}-\phi_{i}\left(z_{s}\right)\right) f_{s}\right\| \underset{s \rightarrow \infty}{\longrightarrow} 0 \text { for } i=1, \cdots, p .
$$

But $\lambda \notin Z$, so there is $\delta^{1}>0$ such that for every $s=1,2, \cdots$, $\left|\operatorname{det}\left(\lambda_{i_{s}}-\phi_{i_{s}}\left(z_{s}\right)\right)\right| \geq \delta^{1}$, (for simplicity we will write further $i_{s}=i_{0}$ ).

The last inequality guarantees existence of $\left(\lambda_{i_{0}}-\phi_{i_{0}}\left(z_{k}\right)\right)^{-1}$. Note next that

$$
\left\|\left(\lambda_{i_{0}}-\phi_{i_{0}}\left(z_{k}\right)\right)\right\|_{L\left(C^{n}\right)}<M \text { for certain } M \text { and } k=1,2, \cdots
$$

Indeed, for an element $a_{i j}\left(z_{k}\right)$ of the inverse matrix $\left(\lambda_{i_{0}}-\phi_{i_{0}}\left(z_{k}\right)\right)^{-1}$ we have equality:

$$
\left|\alpha_{i j}\left(z_{k}\right)\right|=\left|M_{j i}\left(z_{k}\right)\right| /\left|\operatorname{det}\left(\lambda_{i_{0}}-\phi_{i_{0}}\left(z_{k}\right)\right)\right| \leq K / \delta^{1}<\infty
$$

where $M_{j i}$ denotes the cofactor of degree $n-1$ of the matrix $\left(\lambda_{i_{0}}-\phi_{i_{0}}\left(z_{k}\right)\right)$. Hence (2) is satisfied.

Comparing (2) and (1) we obtain

$$
\begin{aligned}
1 & =\left\|f_{k}\right\|=\left\|\left(\lambda_{i_{0}}-\phi_{i_{0}}\left(z_{k}\right)\right)^{-1}\left(\lambda_{i_{0}}-\phi_{i_{0}}\left(z_{k}\right)\right) f_{k}\right\| \\
& \leq M \cdot\left\|\left(\lambda_{i_{0}}-\phi_{i_{0}}\left(z_{k}\right)\right) f_{k}\right\| \rightarrow 0 .
\end{aligned}
$$

This contradiction proves ( $\alpha$ ). Now we can apply a theorem of Fuhrmann [6] and obtain a system of matrices $\psi_{i} \in H_{n \times n}^{\infty}$ such that

$$
\sum_{i=1}^{p} \hat{T}_{\psi_{i}}\left(\lambda_{i}-\hat{T}_{\phi_{i}}\right)=\mathcal{T}
$$

Note next that for certain $\eta>0$,

$$
\inf _{\Delta} \sum_{i=1}^{p}\left|\operatorname{det}\left(\bar{\lambda}_{i}-\phi_{i}(z)^{*}\right)\right| \geq \eta \quad\left(\text { because } \operatorname{det} A^{*}=\overline{\operatorname{det} A}\right)
$$

and this inequality implies, as before,

$$
\inf _{\Delta}\left\{\sum_{i=1}^{p}\left\|\left(\bar{\lambda}_{i}-\phi_{i}(z)^{*}\right) f\right\|, f \in \mathbf{C}^{n},\|f\|=1\right\}>0 .
$$

Using the Fuhrmann theorem once again, we obtain a system of matrices $\theta_{i} \in H_{n \times n}^{\infty}$ such that

$$
\sum_{i=1}^{p}\left(\lambda_{i}-\hat{T}_{\phi_{i}}\right) \hat{T}_{\theta_{i}}=\mathcal{T}
$$


The equalities (3) and (4) yield the desired inclusion.

Remark 2. The inclusion in Theorem 1 cannot be replaced by the equality, but if $p=1$, the equality holds true [8].

2. Now we will find the joint approximate point spectrum of Wiener-Hopf operators on compact abelian groups.

Let $G$ be a locally compact abelian group with dual group $\hat{G}$ and let $\Sigma$ denote a fixed subsemigroup of $\hat{G}$ which is a borel subset of $\hat{G}$. Denote by $\mu$ and $\hat{\mu}$ the normalized Haar measures on $G$ and $\hat{G}$, respectively, and by $\mathfrak{Q}^{2}(G)$ and $\mathfrak{\complement}^{2}(\hat{G})$, as usual, the Hilbert spaces of square integrable complex functions. Let $\mathcal{F}$ be the Fourier transform $\mathfrak{Q}^{2}(G)$ onto $\mathfrak{Q}^{2}(\hat{G})$. Denote by $H^{2}$ the subspace of $\mathfrak{L}^{2}(G)$ consisting of the functions $f$, for which support $\mathfrak{F}_{f} \subset \Sigma$.

If $\varphi$ is a bounded measurable function on $G$, then we define the linear operators $L_{\varphi}$ and $W_{\varphi}$ on $\mathcal{\complement}^{2}(G)$ and $H^{2}$ by

$$
L_{\boldsymbol{\varphi}} f=\boldsymbol{\varphi} f, \quad W_{\boldsymbol{\varphi}} h=P(\boldsymbol{\varphi} \cdot h) .
$$

where $P: \varrho^{2}(G) \rightarrow H^{2}$ is a projection.

We shall call $W_{\varphi}$ a Wiener-Hopf operator. Let $\phi=\left(\varphi_{1}, \cdots, \varphi_{p}\right)$ be $p \cdot$ tuples of functions in $\mathscr{L}^{\infty}(\mu)$. We will denote by $R(\phi)$ the set of all $\left(z_{1}, \cdots\right.$, $\left.z_{p}\right) \in \mathbf{C}^{p}$ such that for every $\epsilon>0$

$$
\mu\left\{g \in G, \sum_{i=1}^{p}\left|\boldsymbol{\varphi}_{i}(g)-z_{i}\right|<\epsilon\right\}>0 .
$$

Define also the weak ${ }^{*}$-closed subalgebra of $\varrho^{\infty}(\mu)$ of all functions $\varphi$ such that $L_{\varphi} H^{2} \subset H^{2}$. Denote it by $H^{\infty}$. Let $A$ be the subalgebra of all continuous functions of $H^{\infty}$. For $G=\Gamma$, Dash proved [4] the inclusion

$$
\sigma_{\pi}\left(L_{\varphi_{1}}, \cdots, L_{\varphi_{p}}\right)=R(\phi) \subseteq \sigma_{\pi}\left(T_{\varphi_{1}}, \cdots, T_{\varphi_{p}}\right) .
$$

Following Coburn and Douglas [3] assume now that $\hat{\mu}(\Sigma)>0$ and $\Sigma$ generates $\hat{G}$. Then the nets of operators $\left\{B_{\alpha i}\right\}_{\alpha \in \Sigma}, i=1, \cdots, p$, defined on $\mathfrak{L}^{2}(G)$ by $B_{a i}=L_{\alpha}^{*} W_{\varphi_{i}} P L_{a}$ are strongly convergent to $L_{\boldsymbol{\varphi}_{i}}$ (for proof consult [3]) and the proof of Dash works in this general setting. Let $H^{\infty}+C(G)$ be the linear space of all functions of the form $\varphi+\psi$, where $\varphi \in H^{\infty}$ and $\psi \in C(G)$. Now we will prove

Theorem 2. Let $G$ be a compact abelian group. Assume that $A$ is a Dirichlet algebra. Let $\phi=\left(\boldsymbol{\varphi}_{1}, \cdots, \varphi_{p}\right)$ be p-tuples of real functions in $\overline{H^{\infty}+C(G)}$ (closed linear span). Then we have equality 


$$
\sigma_{\pi}\left(w_{\varphi_{1}}, \cdots, W_{\varphi_{p}}\right)=R(\phi) .
$$

Proof. By the remark preceding the theorem, the inclusion $R(\phi) \subseteq$ $\sigma_{\pi}\left(W_{\varphi_{1}}, \cdots, W_{\varphi_{p}}\right)$ is true.

Now we will prove the inverse inclusion. Suppose it does not hold. Then there is a point $\left(\lambda_{1}, \cdots, \lambda_{p}\right) \notin R(\phi)$ such that $\left\|\lambda_{i} f_{k}-P\left(\varphi_{i} \cdot f_{k}\right)\right\| \rightarrow 0$ $(k \rightarrow \infty), i=1, \cdots, p$, for certain sequence $\left\{f_{k}\right\}$ of vectors in $H^{2}$. Assume first that $\boldsymbol{\varphi}_{i}=\psi_{i}+\theta_{i}$ where $\psi_{i} \in H^{\infty}, \theta_{i} \in C(G)$ and $\operatorname{Re} \theta_{i}=\operatorname{Re} \eta_{i}$ for certain $\eta_{i} \in A$. It follows that

$$
\left(\left(\psi_{i}+\theta_{i}-\lambda_{i}\right) f_{k}, \psi_{i} f_{k}\right) \underset{k \rightarrow \infty}{\longrightarrow} 0, \quad i=1, \cdots, p
$$

and

$$
\left.\left(\left(\psi_{i}+\theta_{i}-\lambda_{i}\right) f_{k}, \bar{\psi}_{i} f_{k}\right) \underset{k \rightarrow \infty}{\longrightarrow} 0, \quad i=1, \cdots, p \quad \text { (because } \lambda_{i} \in \mathbf{R}\right) .
$$

Similary

and

$$
\left(\left(\psi_{i}+\theta_{i}-\lambda_{i}\right) f_{k}, \eta_{i} f_{k}\right) \underset{k \rightarrow \infty}{\longrightarrow} 0
$$

$$
\left(\left(\psi_{i}+\theta_{i}-\lambda_{i}\right) f_{k}, \vec{\eta}_{i} f_{k}\right) \underset{k \rightarrow \infty}{\longrightarrow}, \quad i=1, \cdots, p .
$$

Consequently we have

$$
\begin{aligned}
\|\left(\psi_{i}\right. & \left.+\theta_{i}-\lambda_{i}\right) f_{k} \|^{2} \\
& =\left(\left(\psi_{i}+\theta_{i}-\lambda_{i}\right) f_{k},\left(\operatorname{Re} \psi_{i}+\operatorname{Re} \eta_{i}-\lambda_{i}\right) f_{k}\right) \underset{k \rightarrow \infty}{\longrightarrow} 0, i=1, \cdots, p .
\end{aligned}
$$

Summing for $i=1, \cdots, p$ we obtain

$$
\int_{G} \sum_{i=1}^{p}\left(\psi_{i}+\theta_{i}-\lambda_{i}\right){ }^{2}\left|f_{k}\right|^{2} d \mu \underset{k \rightarrow \infty}{\longrightarrow} 0
$$

but $\left(\lambda_{1}, \cdots, \lambda_{p}\right) \notin R(\phi)$, a contradiction. If $\operatorname{Re} \theta_{i} \in C_{R}(G)$ are arbitrary then we can choose sequences $\left\{\eta_{i s}\right\} \subset A$ such that $\operatorname{Re} \eta_{i s} \rightrightarrows \operatorname{Re} \theta_{i}, i=$ $1, \cdots, p$. By the same reasoning as before we also get a contradiction. For arbitrary $\boldsymbol{\varphi}_{i} \in \overline{H^{\infty}+C(G)}$, let $\boldsymbol{\varphi}_{i k} \in H^{\infty}+C(G)$ and $\boldsymbol{\varphi}_{i k} \rightrightarrows \boldsymbol{\varphi}_{i}$. Note that if $\left(\lambda_{1}, \cdots, \lambda_{p}\right) \notin R(\phi)$ then $\left(\lambda_{1}, \cdots, \lambda_{p}\right) \notin R\left(\phi_{k}\right)$ for $k \geq k_{0}$, where $\phi_{k}=$ $\left(\varphi_{1 k}, \cdots, \varphi_{p k}\right)$ and the proof follows immediately.

Remark 3. Theorem 2 is also true for $p$-tuples of arbitaray $\boldsymbol{\varphi}_{i} \in H^{\infty}$ without the assumption that $A$ is a Dirichlet algebra.

Remark 4. When $p=1$ and $G=\Gamma$ Theorem 2 has been proved by Klein $[7]$.

\section{REFERENCES}

1. F. Bonsall and J. Duncan, Numerical ranges of operators on normed spaces 
and of elements of normed algebras, London Math. Soc. Lecture Note Series, 2, Cambridge Univ. Press, New York and London, 1971. MR 44 \#5779.

2. J. Bunce, The joint spectrum of commuting nonnormal operators, Proc. Amer. Math. Soc. 29 (1971), 499-505. MR 44 \#832.

3. L. A. Coburn and R. G. Douglas, On $C^{*}$-algebras of operators on a halfspace. I, Inst。 Hautes Études Sci. Publ. Math. No. 40 (1972).

4. A. T. Dash, Joint spectra, Studia Math. 45 (1973), 225-237.

5. R. G. Douglas, Banach algebra techniques in operator theory, Academic Press, New York, 1972.

6. P. A. Fuhrmann, On the corona theorem and its application to spectral problems in Hilbert space, Trans. Amer. Math. Soc. 132 (1968), 55-66. MR 36 \#5751.

7. E. M. Klein, Algebraic properties of Toeplitz operators, Math. Ann. 202 (1973), 203-207.

8. H. R. Pousson, Systems of Toeplitz operators on $H^{2}$, Proc. Amer. Math. Soc. 19 (1968), 603-608. MR 37 \#783.

9. J. L. Taylor, A joint spectrum for several commuting operators, J. Functional Analysis 6 (1970), 172-191. MR 42 \#3603.

INSTYTUT MATEMATYCZNY PAN, KRAKÓW, SOLSKIEGO 30, POLAND 